\title{
Les affinités taxonomiques de Microsporum ferrugineum отА, 1922 appréciées par l'analyse immunoélectrophorétique de sa structure antigénique
}

\author{
par Jean BIGUET, Suzanne ANDRIEU et Bernard LALOUX
}

En 1922, Ota décrit un Dermatophyte nouveau, agent de teigne tondante du cuir chevelu et de la peau glabre en Mandchourie; la lésion clinique du cheveu est celle d'une microsporie. Son aspect microscopique est très pauvre : pas de macro ni de microconidies, des filaments généralement faviformes et formant des chlamydospores. La colonie est de croissance très lente, la couleur de la culture rouille à jaune ocre. Le nom de Microsporum ferrugineum que lui donne Ota tient compte de ce caractère.

Depuis sa description, ce Dermatophyte a subi un certain nombre de vicissitudes génériques dont voici les principales:

Il passe dès 1923 dans le genre Grubyella créé par Ota et Langeron où il voisine avec Achorion schoenleini et des Trichophyton ectothrix mégasporés (T. album, $T$. discoides, T. ochraceum), Guiart et Grigorakis le transportent dans le genre Achorion en 1928 et Paldrock (1953) ira encore plus loin dans cette voie en l'assimilant purement et simplement à $A$. schoenleini.

Cependant pour la majorité des auteurs modernes, après Langeron et Milochevitch (1930), il sera classé dans les Trichophyton: cet avis sera, en effet, partagé par Talice (1931), puis par Emmons (1934). Ce dernier auteur, se basant uniquement sur le caractère botanique, révise la classification des Dermatophytes et fait tomber en synonymie le genre Achorion devant Trichophyton. Conant (1945), suivant Emmons, s'inspirera également de Langeron et coll. (1930) pour placer côte à côte dans les Trichophyton faviformes $M$. ferrugineum et $A$. schoenleini.

Enfin, Vanbreuseghem, en 1950, après une étude très soigneuse de 136 souches africaines de $M$. ferrugineum conclut que ce parasite est indiscutablement un Trichophyton malgré la nature microsporique de la lésion qu'il détermine dans le cheveu.

Contre cette conception qui fait du Dermatophyte d'Ota (1922) un Trichophyton, il y a peu d'opposants: Dodge cependant en 1936, l'appelle encore Microsporum ferrugineum. Mais c'est Rivalier qui, en 1954, critiquera avec le plus d'énergie l'appartenance de ce Champignon au genre Trichophyton. Il reste profondément convaincu de son appartenance au genre Microsporum [à l'époque, suivant une suggestion de

* Travail effectué avec le concours financier de l'Institut national de la Santé et de la Recherche médicale. 
Langeron (1930), il écrit Sabouraudites] dont il représenterait l'espèce la plus dégradée. Il appuie son opinion sur le fait : a) que le regroupement dans un même genre d'espèces stériles et d'espèces fertiles a déjà été accepté par les mycologistes en ce qui concerne le genre Trichophyton; $b$ ) que parmi les nombreuses formes culturales de $M$. ferrugineum, il en existe qui constituent un passage insensible vers $M$. audouini ; c) que durant sa vie parasitaire, $M$. ferrugineum se comporte au niveau du poil comme un Microsporum classique. Il conclut : «J'incline même à croire que $S$. audouini et $S$. ferrugineum sont deux formes adaptatives issues d'une souche commune qui est peut-être Sabouraudites canis. » En fait, cette opinion se trouve renforcée par les travaux de quelques auteurs russes qui auraient réussi à induire la transformation de $M$. ferrugineum en $M$. lanosum (Sigalova, 1951) ou en une variété voisine de cette espèce (Zimerinov et coll., 1953) *

En 1960, Biguet et coll. refusent d'identifier $T$. ferrugineum à $T$. soudanense contrairement à l'avis des auteurs portugais (Salazar Leite et coll., 1950) et estiment que l'opinion de Rivalier concernant la systématique de $T$. ferrugineum mérite un examen approfondi. Ils suggèrent de tenter de résoudre les prob'èmes taxonomiques en suspens, et plus particulièrement l'appartenance générique de ce Dermatophyte par l'analyse immunoélectrophorétique comparée des structures antigéniques.

L'année suivante, ces auteurs démontrent que les techniques de précipitation en gélose sont effectivement applicables au dénombrement des fractions antigéniques des Dermatophytes (Biguet et coll., 1961) et ils s'emploient par la suite à l'étude comparée des structures antigéniques de ce groupe de Champignons. Le présent travail relate les résultats obtenus concernant Microsporum ferrugineum.

\section{I. - Matériel et Méthodes}

Les Dermatophytes suivants sont utilisés :

- M. ferrugineum; $M$. canis et $M$. audouini desquels dériverait peut-être l'espèce précédente ;

- T. violaceum et $T$. rubrum: deux Trichophyton authentiques très différents par les lésions qu'ils provoquent et leur localisation ;

- T. soudanense dont la morphologie des colonies est très voisine de $M$. ferrugineum ** ;

- T. schoenleini souvent rapproché dans les classifications modernes du Champignon d'OTA ;

- enfin T. mentagrophytes (forme culturale granulosum) qui est, contrairement aux -autres Trichophyton choisis, une espèce à morphologie microscopique riche.

a) Les souches purifiées des Dermatophytes sont cultivées sur milieu de Sabouraud liquide; le mycélium est récolté après un mois et demi de développement. Les antigènes sont obtenus par broyage au mortier glace et extraction par le sérum salé $(0,05 \mathrm{M})$ dialysés, puis lyophilisés.

* Zimerinov et coll. ont cultivé $M$. ferrugineum trois mois et demi dans six filtrats de culture de $M$. canis. Nous n'avons malheureusement pas réussi à nous procurer leur texte, ni celui de Sigalova. Leurs travaux sont cités par Troyan en 1958 dans une note mal.eureusement dépourvue de bibliographie.

**: Vanbreuseghem a toutefois insisté récemment (1963) sur les différences de la morphologie macroscopique des deux espèces cultivées sur 25 milieux différents. 
b) Les antisérums de lapin sont préparés par injections hebdomadaires sous-cutanées (méthode de Freund) d'antigène lyophilisé $(5 \mathrm{mg})$, additionné de Champignon entier sec $(20 \mathrm{mg})$. Les sérums sont testés par la méthode d'Ouchterlony et sont, en général, utilisables au bout de quatre à cinq mois d'immunisation.

c) L'immunoélectrophorèse est conduite en agarose (tampon véronal sodé, $\mathrm{pH} 82, \mathrm{f}$. i. : $0,1)$. Après séparation électrophorétique des extraits antigéniques effectuée pendant 5 heures 30 sous une différence de potentiel de 20 volts aux extrémités des lames $(11 \times 5 \mathrm{~cm})$, les diagrammes sont révélés par les antisérums de lapin.

\section{II. - Résultats et Commentaires}

L'essentiel de nos résultats est schématisé dans les figures I et II et résumé dans le tableau I.

Tableau I : Les réactions croisées de huit Dermatophytes appréciées par deux antisérums:

M. ferrugineum et M. canis

\begin{tabular}{|c|c|c|c|c|c|c|c|c|}
\hline \multirow[b]{2}{*}{ ANTISÉRUMS } & \multicolumn{8}{|c|}{ ANTIGÈNES } \\
\hline & 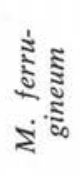 & 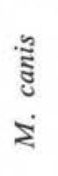 & 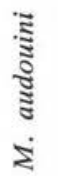 & 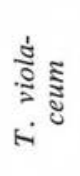 & 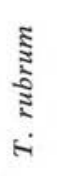 & 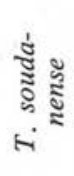 & 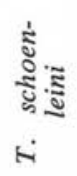 & 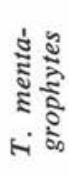 \\
\hline M. ferrugineum & 15 & 16 & 14 & 6 & 6 & 5 & 6 & 9 \\
\hline M. canis & 14 & 20 & 13 & 7 & 8 & 7 & 7 & 9 \\
\hline
\end{tabular}

Il faut d'abord préciser que la qualité des antigènes utilisés a été appréciée par leur confrontation contre l'antisérum homologue. C'est ainsi que les antigènes $M$. audouini, $T$. violaceum, $T$. rubrum et $T$. mentagrophytes précipitent 16 arcs contre les immunsérums de lapins correspondants et $T$. schoenleini 14 arcs. Par contre, l'antigène $T$. soudanense semble de qualité médiocre puisqu'il ne précipite que 10 arcs dans les mêmes conditions. Quant à l'antigène $M$. ferrugineum lui-même, contrairement à l'antigène $M$. canis qui est excellent (20 arcs de précipitation), nous n'avons pas réussi à l'obtenir de qualité parfaite, ceci en dépit de multiples essais réalisés dans des conditions d'extraction variables sur de multiples souches. La preuve en est apportée par le fait que l'antisérum $M$. ferrugineum réagit légèrement mieux contre l'espèce hétérologue $M$. canis (16 arcs au lieu de 15). C'est vraisemblablement cette qualité médiocre de l'antigène $M$. ferrugineum qui explique encore que son application contre l'antisérum $M$. canis ne révèle que 14 arcs au lieu de 16 . Notons toutefois que les réactions inverses n'aboutissent pas toujours, même avec un excellent matériel, à des résultats absolument identiques ; les précipitations ne peuvent se produire, en effet, que 


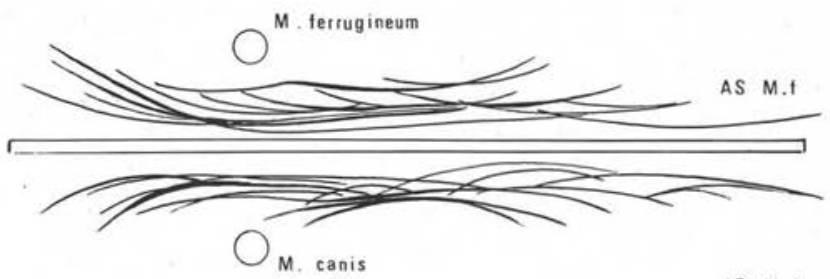

AS M. $\mathrm{I}$

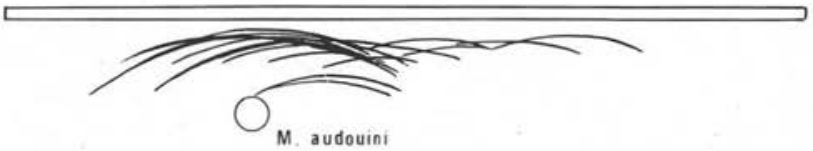

AS M.i.

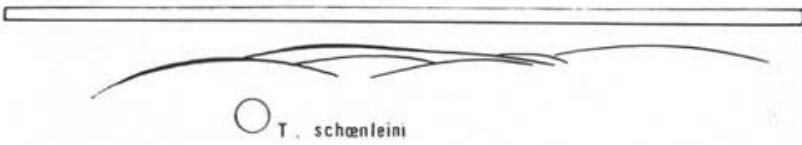

AS M.f

AS M.f

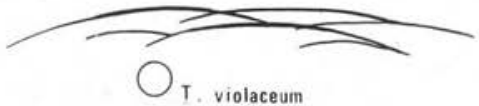

AS M. $\mathrm{f}$

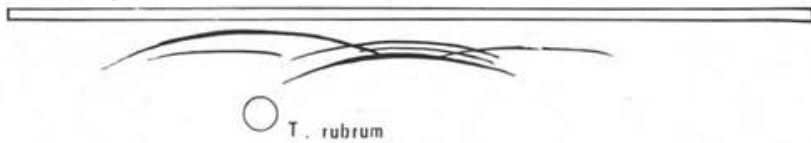

Antisérum M.ferrugineum

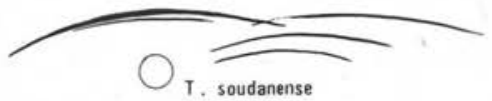

Fig. 1. - Analyse immunoélectrophorétique comparée des structures antigéniques de $M$. ferrugineum; $M$. canis, $M$. audouini, $T$. schoenleini, $T$. mentagrophytes, $T$. violaceum, $T$. rubrum et $T$. soudanense révélées par l'antisérum $M$. ferrugineum 
dans certaines limites du rapport quantitatif antigène-anticorps. Si l'antigène et l'anticorps ont des composants multiples et si l'on ne peut modifier que les quantités globales d'antigène et d'anticorps confrontées, on conçoit que leurs variations quantitatives de structure retentissent plus ou moins favorablement sur le nombre d'arcs de l'immunoélectrophorégramme.

Ces remarques et ces réserves faites, la lecture de notre tableau I et l'examen des figures I et II font apparaître que les structures antigéniques de $M$. ferrugineum sont nettement plus apparentées aux Microsporum qu'aux Trichophyton. On constate, en effet, qu'il existe entre $M$. ferrugineum et les deux espèces $M$. canis et $M$. audouini une communauté antigénique chiffrée respectivement par 16 et 14 arcs. Cette communauté s'étage entre cinq et six arcs seulement lorsque le Dermatophyte d'Ota est comparé à $T$. soudanense, $T$. violaceum, $T$. rubrum et $T$. schoenleini. Elle atteint un maximum de neuf lorsque cette comparaison est réalisée avec $T$. mentagrophytes qui, sous sa variété culturale granuleuse, se distingue nettement des autres Trichophyton par la richesse de ses formes de reproduction.

Notons encore que $T$. mentagrophytes possède également 9 fractions communes avec $M$. canis; l'analyse antigénique montre donc qu'il n'existe pas de différences tranchées entre les genres Trichophyton et Microsporum; elle confirme l'homogénéité du groupe des Dermatophytes.

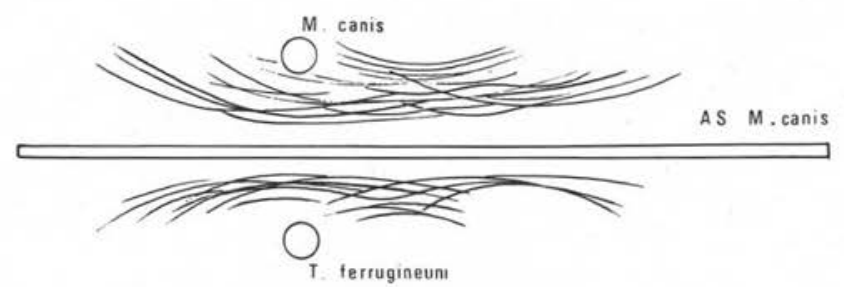

Fig. 2. - Analyse immunoélectrophorétique comparée des structures antigéniques de $M$. canis et $M$. ferrugineum révélées par l'antisérum $M$. canis

\section{III. - Conclusions}

L'analyse comparée de la mosaïque antigénique du Dermatophyte décrit par Ota en 1922 sous le nom de Microsporum ferrugineum et de sept autres espèces appartenant aux genres Microsporum ( $M$. audouini, $M$. canis) et Trichophyton ( $T$. violaceum, $T$. rubrum, $T$. soudanense, $T$. schoenleini, $T$. mentagrophytes) conduit à cette conclusion que le Champignon d'Ota est immunologiquement beaucoup plus proche des Microsporum que des Trichophyton. Sans considérer l'argument antigénique comme définitif dans le cadre d'un groupe aussi homogène que celui des Dermatophytes, il nous paraît cependant supérieur à des arguments botaniques qui reposent essentiellement non pas sur la présence de structures caractéristiques, mais au contraire sur l'absence de telles structures. 
Jusqu'à preuve du contraire, et suivant en ceci l'avis de Rivalier (1954), nous tenons désormais le Dermatophyte décrit par Ota pour un Microsporum morphologiquement dégradé.

\section{Bibliographie}

Biguet (J.), Andrieu (S.), Tran Van Ky (P.), 1961. - Application des méthodes électrophorétiques et immunochimiques à l'étude des fractions antigéniques des Dermatophytes. Premiers résultats concernant Ctenomyces mentagrophytes. C.R. Acad. Sci., Fr., 253, $2,167$.

Biguet (J.), Cochet (G.), Deblok (S.), Andrieu (S.), Duc (G.), 1960. — Les affinités taxonomiques de quelques Trichophyton africains agents de tondantes microsporiques ou trichophytiques. Ann. Parasitol. hum. comp., Fr., 35, 409.

Conant (N. F.), Smith (D. T.), Baker (R. D.), Callaway (J. L.), et Martin (D. S.). - Manual of clinical mycology, $1^{\text {re }}$ édit., 1945, $2^{\text {e }}$ édit., 1954, W. B. Saunders Company édit., Philadelphie et Londres.

Dodge (C. W.), 1936. - Medical Mycology, Henri Kimpton, edit., Londres.

Emmons (C. W.), 1934. - Dermatophytes. Natural grouping based on the form of the spores and accessory organs. Arch. Dermatol, Syph., 30, 337.

Guiart (J.) et Grigorakis (L.), 1928. - La classification botanique des Champignons des teignes. Lyon médical, 141, 369.

Langeron (M.) et Milochevitch (S.), 1930. - Morphologie des Dermatophytes. Ann. Parasitol hum. comp., Fr., 8, 465.

OтA (M.), 1922. - Sur deux espèces nouvelles de Dermatophytes en Mandchourie: Microsporum ferrugineum et Trichophyton pedis n. sp. Bull. Soc. Path. Exot., 15, 588. (Jap. J. Derm. Urol., 1921, 21, 201).

Ota (M.) et Langeron (M.), 1923. - Nouvelle classification des Dermatophytes. Ann. Parasitol. hum. comp., Fr., 1, 305.

Paldrock (H.), 1953. - On the variability and classification of Dermatophytes. Acta Dermato. Vernerol., 3, 1.

Rivalier (E.), 1954. - Description de Sabouraudites praecox nov. sp., suivie de remarques sur le genre Sabouraudites. Ann. Inst. Past., Paris, 86, 276.

Salazar Leite (A.) et Bastos Da LuZ (I. V.), 1950. - Estudos sobre os agentes causais de tinhas en nativos africanos de raça negro. II. Congresso Luso Espanhol de Dermatologia, Lisbonna.

Sigalova (E. E.), 1951. - In Troyan (G. A.), 1958.

TALICE (R. V.), 1931. - Sur une souche de Trichophyton ferrugineum (Ota, 1921) (Microsporum ferrugineum Ota, 1921) isolée à Montevideo. Ann. Parasitol. hum. comp., 9, 77.

Troyan (G. A.), 1956. - Variabilité dirigée des Dermatophytes. Mikrobiol. Journ. Acad. Nank., Rep. Soc. Sov. Ukrain., 20, 21 (en Ukrainien).

VANBreuseghem (R.), 1950. - Etude de 136 souches de $T$. ferrugineum (Ota, 1921) LANGERON et Milochevitch, 1930 et de sa variété blanche isolée au Congo Belge. Ann. Parasitol., 25, 485.

VANBREUSEGHEM (R.), 1963. - Contribution à l'identification de Trichophyton (Langeronia) soudanense et du Trychophyton ferrugineum. Ann. soc., Belge Med. Trop., 3, 259.

Zimerinov (A. A.), Besnos (T. I.) et Rafalovitch (C. M.), 1953. - In Troyan (G. A.), 1958.

(Laboratoire de Parasitologie de la Faculté de Médecine et de Pharmacie de Lille Dir. : Pr J. BIGUET) 